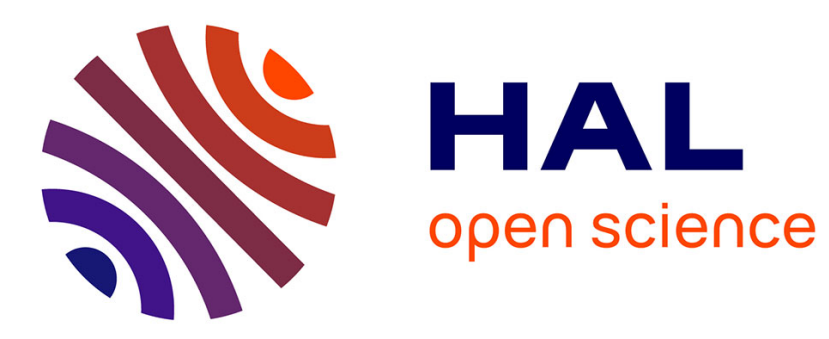

\title{
Structure from Motion with Variable Focal Length: Interconnected Fuzzy Observer
}

Rayane Benyoucef, Hicham Hadj-Abdelkader, Lamri Nehaoua, Hichem Arioui

\section{To cite this version:}

Rayane Benyoucef, Hicham Hadj-Abdelkader, Lamri Nehaoua, Hichem Arioui. Structure from Motion with Variable Focal Length: Interconnected Fuzzy Observer. 60th IEEE conference on Decision and Control (CDC 2021), Dec 2021, Austin, Texas, United States. pp.6145-6150, 10.1109/CDC45484.2021.9683139 . hal-03484304

\section{HAL Id: hal-03484304 https://hal.science/hal-03484304}

Submitted on 17 Dec 2021

HAL is a multi-disciplinary open access archive for the deposit and dissemination of scientific research documents, whether they are published or not. The documents may come from teaching and research institutions in France or abroad, or from public or private research centers.
L'archive ouverte pluridisciplinaire HAL, est destinée au dépôt et à la diffusion de documents scientifiques de niveau recherche, publiés ou non, émanant des établissements d'enseignement et de recherche français ou étrangers, des laboratoires publics ou privés. 


\title{
Structure from Motion with Variable Focal Length: Interconnected Fuzzy Observer
}

\author{
R. Benyoucef, H. Hadj-Abdelkader, L. Nehaoua and H. Arioui
}

\begin{abstract}
In this paper, a 3D point feature depth and camera focal length estimation is proposed, using a partially calibrated low cost monocular camera. The camera intrinsic parameters are known, except for the focal length, which may vary across different views. The camera perspective projection model is augmented using dynamic extension approach, then decomposed into two interconnected subsystems. The subsystems are described as quasi-Linear Parameter Varying (qLPV) systems with unmeasured premise variables for which an Interconnected Fuzzy Observer (IFO) is designed. Necessary and sufficient conditions to ensure the observer existence are presented. The error convergence analysis is performed based on Lyapunov theory associated with Lipchitz condition. Gains that guarantee the asymptotic stability of the estimation error are computed in terms of Linear Matrix Inequalities (LMI) with eigenvalues clustering in LMI region to improve the estimation performance.
\end{abstract}

Keywords: Nonlinear Luenberger Observer, quasi-LPV Systems, Camera Auto-calibration, Structure from Motion, LMI constraints, Lyapunov Theory

\section{INTRODUCTION}

Camera images provide a large amount of data. Estimating the data contained in images is a challenge that extends through many disciplines. Recovering depth information using a moving camera can be used to estimate the range (and hence, a 3D structure) of the feature points from 2D images which is usually referred to as Structure from Motion (SfM). This problem has been the central focus in computer vision community, motivating a large number of research work. Researchers in the field have proposed several solutions exploiting filtering tools as in [12] where the problem of SfM is addressed using a finite-dimensional approximation to the optimal filter. Also, in [2] an Unscented Kalman Filter is implemented for depth estimation and in [1], the authors implemented an extended Kalman filter to solve the $3 \mathrm{D}$ reconstruction problem. These tools have proved to be efficient, however, they assume linearized structures and lack a convergence guarantee. Besides, such solutions necessitate prior knowledge of the noise. Accordingly, other approaches propose deterministic nonlinear observation based framework. For instance in [3], an active structure from motion scheme of a feature point is addressed by optimizing the convergence rate of the estimation error to impose a desired transient response. And along similar lines, application to include other geometrical primitives as lines [4], spherical and cylindrical targets [5] and moments [6] were introduced.

All authors are with IBISC Lab, Univ. Evry, University of ParisSaclay University, 40 Rue du Pelvoux, 91080 Courcouronnes. France rayane.benyoucef@univ-evry.fr
However, the observer synthesis of all the previously cited works is based on neglecting a disturbance term which may have a big impact on the state estimation.

The vast majority of existing approaches assume that the camera is calibrated beforehand, such that all the intrinsic parameters remain constant across various views. This can be very restrictive since it excludes adjusting the focal length to zoom or focus.

The camera calibration plays an essential role in the accuracy of the scene reconstruction task. Many works, as in [7], have demonstrated that uncertainties in camera calibration parameters will affect the accuracy of 3D reconstruction. Furthermore, An analysis of the impact of the assumptions on camera intrinsic parameters upon the scene structure and camera motion parameters is discussed in [9]. In [13], it has been shown that an accurate focal length calculation is considered to be the most important components in the camera calibration model when calculating surface point coordinates via triangulation. This led to an intensive research on the possibility to obtain the Euclidean 3D reconstruction without using a fully calibrated camera. Among these research works, a few handled the problem of estimating the unknown focal length in the context of 3D reconstruction. One can cite [10] where a method for incremental Non-Rigid Structure-fromMotion (NRSfM) with unknown focal length is presented by employing a searching scheme that checks for the focal length preserving the highest possible isometric reconstruction accuracy across views. Moreover, in [11], the authors proposed a method to reconstruct 3D scene from collection of images with known (in principle single image with known focal length) and unknown focal lengths, by providing a minimal solution to finding the relative pose between a fully calibrated camera and a camera with an undefined focal length. This paper extends our previous work proposed in [8], that solves the problem of 3D structure reconstruction, assuming a precise knowledge of the camera calibration parameters. In this work we investigate the depth reconstruction of a feature point observed by a partially calibrated camera with varying focal length assuming square-shaped pixels. The main idea is to immerse the camera perspective model into an augmented system in order to obtain an appropriate structure to design the Interconnected Fuzzy Observer (IFO). The augmented system is decomposed into two interconnected quasi-Linear Parameter Varying (qLPV) systems. Both submodels are transformed into Takagi-Sugeno (T-S) form with unmeasurable premise variable. An IFO is designed in order to estimate the depth of the feature point and the camera focal length. The stability of the observer is investigated 
based on Lyapunov theory and sufficient conditions for the convergence of the estimation error are established using Linear Matrix Inequality (LMI) formalism. The paper is organized as follows: Section II recalls basic definition and notations to be used throughout the paper and describes the vision system model. In section III, the quasi-LPV system is described and conditions to ensure the observer existence are discussed. While section IV addresses the design of the non-linear observer to estimate the unknown depth and the camera focal length. Simulation results using synthetic data are provided in section IV, followed by conclusions in section $\mathrm{V}$.

\section{BACKGROUND}

Before formulating the problem, notations, and basic lemmas are given to be used later to prove the main results.

\section{A. Notations and basic definitions}

In the sequel, matrices are represented in upper case bold letters $\mathbf{X}$ and vectors in lower case bold letters $\mathbf{x} . \mathbf{X}=\mathbf{X}^{\top}$ is the definition of the symmetric matrix. $(.)^{-1}$ is the inverse of a given square matrix. $\mathbf{X}>0($ resp. $\mathbf{X}<0)$ denotes that $\mathbf{X}$ is a definite positive (resp. negative) matrix. $\mathbf{O}$ and $\mathbf{I}$ refers to zero and identity matrices with appropriate dimensions. $[.]_{\times}$denotes the skew-symmetric matrix of a given vector and $\|()$.$\| represents the Euclidean norm. And \mathfrak{R}$ denotes the real part of a given complex number and finally, to simplify notation, we omit function arguments when possible.

Lemma 1: For every matrix $\mathbf{G}=\mathbf{G}^{T}>0, \mathbf{X}$ and $\mathbf{Y}$ with appropriate dimensions, the property below holds:

$$
\mathbf{X}^{T} \mathbf{Y}+\mathbf{Y}^{T} \mathbf{X} \leq \mathbf{X}^{T} \mathbf{G X}+\mathbf{Y}^{T} \mathbf{G}^{-1} \mathbf{Y}
$$

Lemma 2 (Schur complement lemma): Consider the following convex nonlinear inequalities:

$$
\mathbf{R}>0, \quad \mathbf{T}-\mathbf{S R}^{-1} \mathbf{S}^{T}>0
$$

where the matrices $\mathbf{T}=\mathbf{T}^{T}, \mathbf{R}=\mathbf{R}^{T}$ and $\mathbf{S}$ are of appropriate dimension. Hence, the previous inequalities can be written in the following form:

$$
\left[\begin{array}{rr}
\mathbf{T} & \mathbf{S} \\
\mathbf{S}^{T} & \mathbf{R}
\end{array}\right]>0
$$

Note that the previous mathematical properties hold for Linear Time Invariant (LTI) systems and also for the case of qLPV systems when considering frozen parameter vectors.

\section{B. Conventional Camera Model}

Let the $3 \mathrm{D}$ point feature $\mathbf{p}$ represented in the camera frame with the coordinates $\mathbf{p}=(X Y Z)^{\top}$. The homogeneous coordinates of its projection onto the image plane is defined by:

$$
\mathbf{m}=\left(\begin{array}{lll}
x & y & 1
\end{array}\right)^{\top}=\left(\begin{array}{lll}
\frac{\lambda}{Z} X & \frac{\lambda}{Z} Y & 1
\end{array}\right)^{\top}
$$

The relative motion of $\mathbf{p}$, as observed in the camera coordinate system, can be expressed by the following kinematics:

$$
\dot{\mathbf{p}}=-v+\mathbf{p} \times \omega=\left(\begin{array}{ll}
-\mathbf{I}_{\mathbf{n}} & {[\mathbf{p}]_{\times}}
\end{array}\right) \mathbf{u}
$$

where $\mathbf{u}=\left(v^{\top} \omega^{\top}\right)^{\top}$ with $v=\left(v_{x} v_{y} v_{z}\right)^{\top}$ and $\omega=$ $\left(\omega_{x} \omega_{y} \omega_{z}\right)^{\top}$ are respectively the instantaneous linear and angular velocities of the origin of the camera expressed in its local frame.

Consequently, one can derive from (4) and (5) the following expression:

$$
\left(\begin{array}{l}
\dot{x} \\
\dot{y}
\end{array}\right)=\left(\begin{array}{cccccc}
-\frac{1}{Z} & 0 & \frac{x}{Z} & x y & -\left(1+x^{2}\right) & y \\
0 & -\frac{1}{Z} & \frac{y}{Z} & \left(1+y^{2}\right) & -x y & -x
\end{array}\right) \mathbf{u}
$$

The transition of the 2D feature point $\mathbf{m}$ from the image coordinates to the pixel coordinates $\mathbf{f}$ is obtained by the following expression:

$$
\mathbf{f}=\left(\begin{array}{lll}
u & v & 1
\end{array}\right)^{\top}=\mathbf{K} \mathbf{m}
$$

where $\mathbf{K}$ represents the calibration matrix that contains the camera intrinsic parameters:

$$
\mathbf{K}=\left(\begin{array}{ccc}
\lambda k_{u} & s & u_{0} \\
0 & \lambda k_{v} & v_{0} \\
0 & 0 & 1
\end{array}\right)
$$

where $\left(u_{0} v_{0}\right)^{\top}$ is the vector representing the pixel coordinates of the principle point, $k_{u}$ and $k_{v}$ are respectively the density of pixels in the horizontal and vertical direction of the pixel axis expressed in number of pixels per millimeter, $s$ is called the skew factor and the focal length $\lambda$ is the distance between the optical center and the image plane expressed in millimeters.

In this paper, we deal with cameras that have orthogonal pixel axis $(s=0)$ and square-shaped pixels $\left(k_{u}=k_{v}=k\right)$. After converting the physical focal length $\lambda$ to pixel unit $(f=\lambda k)$, the camera calibration matrix becomes:

$$
\mathbf{K}=\left(\begin{array}{ccc}
f & 0 & u_{0} \\
0 & f & v_{0} \\
0 & 0 & 1
\end{array}\right)
$$

We assume, in this work, a partially calibrated camera where only the coordinates of the principle point $\left(u_{0} v_{0}\right)$ are known. Taking into account the equation (7) and by defining $f_{u}=$ $u-u_{0}$ and $f_{v}=v-v_{0}$, the expression in (6) takes the following form:

$$
\left(\begin{array}{l}
\dot{f}_{u} \\
f_{v}
\end{array}\right)=\left(\begin{array}{cccccc}
-\frac{f}{Z} & 0 & \frac{f_{u}}{Z} & \frac{f_{u} f_{v}}{f} & -\left(f+\frac{f_{u}^{2}}{f}\right) & f_{v} \\
0 & -\frac{f}{Z} & \frac{f_{v}}{Z} & f+\frac{f_{v}^{2}}{f} & -\frac{f_{u} f_{v}}{f} & -f_{u}
\end{array}\right) \mathbf{u}
$$

In order to estimate the $3 \mathrm{D}$ Euclidean coordinates of the feature point and the camera focal length, we expand the system (10) to include the dynamics of the depth information $\frac{1}{Z}$ denoted by $\chi$ and the focal length $f$.

The dynamics of $\chi$, deduced from the equation (5), is given by:

$$
\dot{\chi}=\left(\begin{array}{llllll}
0 & 0 & \chi^{2} & y \chi & -x \chi & 0
\end{array}\right) \mathbf{u}
$$

The system in (10) is augmented using the dynamic extension approach by generating auxiliary dynamics. This results in a larger new system with relaxed dependency on the unmeasurable premise variables in order to reduce the number of subsystems when considering the TS form. Thus:

$$
\left(\begin{array}{c}
\dot{f}_{u} \\
\dot{f} \\
\dot{\chi} \\
\dot{f}
\end{array}\right)=\left(\begin{array}{cccccc}
-f \chi & 0 & f_{u} \chi & \frac{f_{u} f_{v}}{f} & -\left(f+\frac{f_{u}^{2}}{f}\right) & f_{v} \\
0 & -f \chi & f_{v} \chi & f+\frac{f_{v}^{2}}{\chi} & -\frac{f_{u} f_{v}}{f} & -f_{u} \\
0 & 0 & \chi^{2} & f_{u} \frac{\chi}{f} & -f_{v} \frac{\chi}{f} & 0 \\
0 & 0 & 0 & 0 & 0 & 0
\end{array}\right) \mathbf{u}
$$


Where: $f_{u}$ and $f_{v}$ are the measurable states, computed from the extracted feature point from the image and the depth information $\chi$ and the focal length $f$ are the unmeasurable data to be estimated.

\section{MODEL DESCRIPTION}

The objective of this section is to decompose the system (12) into two suitably defined interconnected systems. Further, we discuss the observer existence conditions that are highly correlated with the way of splitting the system into two subsystems.

\section{A. Quasi-LPV representation}

The system (12) is devided into two sub-models, dynamically coupled, described in qLPV form by:

$$
\begin{aligned}
& \Sigma_{1}\left\{\begin{array}{l}
\dot{\mathbf{x}}=\overline{\mathbf{A}}(\mathbf{x}, \mathbf{z}, \mathbf{u}) \mathbf{x}+\overline{\mathbf{B}}(\mathbf{y}) \omega+\overline{\mathbf{D}}\left(\mathbf{y}_{\mathbf{z}}\right) \mathbf{z} \\
\mathbf{y}_{\mathbf{x}}=\overline{\mathbf{C}} \mathbf{x}
\end{array}\right. \\
& \Sigma_{1}\left\{\begin{array}{l}
\dot{\mathbf{z}}=\breve{\mathbf{A}}(\mathbf{x}, \mathbf{z}, \mathbf{u}) \mathbf{z}+\breve{\mathbf{B}}(\mathbf{y}) \omega+\breve{\mathbf{D}}\left(\mathbf{y}_{\mathbf{x}}\right) \mathbf{x} \\
\mathbf{y}_{\mathbf{z}}=\overline{\mathbf{C}} \mathbf{z}
\end{array}\right.
\end{aligned}
$$

where $\overline{\mathbf{A}}(\mathbf{x}, \mathbf{z}, \mathbf{u}), \breve{\mathbf{A}}(\mathbf{x}, \mathbf{z}, \mathbf{u}) \in \mathbb{R}^{2 \times 2} . \overline{\mathbf{B}}(\mathbf{y}), \breve{\mathbf{B}}(\mathbf{y}) \in \mathbb{R}^{3 \times 2}$ and $\overline{\mathbf{D}}\left(\mathbf{y}_{\mathbf{z}}\right), \overline{\mathbf{D}}\left(\mathbf{y}_{\mathbf{x}}\right) \in \mathbb{R}^{2 \times 2}$. The following vectors $\mathbf{x}, \mathbf{z} \in \mathbb{R}^{2}$, $\omega \in \mathbb{R}^{3}$, and $\mathbf{y}_{\mathbf{x}}, \mathbf{y}_{\mathbf{z}} \in \mathbb{R}$ are respectively the state vectors for the first and the second subsystem, the angular velocity, and the output vectors for the first and the second subsystem.

The matrices defined in the system above are represented as follows:

$$
\begin{gathered}
\overline{\mathbf{A}}(\mathbf{x}, \mathbf{z}, v)=\left(\begin{array}{cc}
\frac{f_{v}}{f} \omega_{x}-\frac{f_{u}}{f} \omega_{y} & \chi v_{x}-\omega_{y} \\
0 & 0
\end{array}\right) \quad \overline{\mathbf{B}}(\mathbf{y})=\left(\begin{array}{ccc}
0 & 0 & f_{v} \\
0 & 0 & 0
\end{array}\right) \\
\breve{\mathbf{A}}(\mathbf{x}, \mathbf{z}, \mathbf{u})=\left(\begin{array}{cc}
\frac{f_{v}}{f} \omega_{x}-\frac{f_{u}}{f} \omega_{y}+\chi v_{z} & -f v_{y} \\
0 & \frac{f_{v}}{f} \omega_{x}-\frac{f_{u}}{f} \omega_{y}+\chi v_{z}
\end{array}\right)
\end{gathered}
$$$$
\overline{\mathbf{D}}(\mathbf{u}, \mathbf{y})=\left(\begin{array}{cc}
0 & f_{u} v_{z} \\
0 & 0
\end{array}\right) \breve{\mathbf{B}}(\mathbf{y})=\left(\begin{array}{ccc}
0 & 0 & -f_{u} \\
0 & 0 & 0
\end{array}\right) \breve{\mathbf{D}}(\mathbf{u})=\left(\begin{array}{cc}
0 & \omega_{x} \\
0 & 0
\end{array}\right)
$$

\section{B. T-S Exact Model}

The subsystems (16) and (14) can be expressed in T-S fuzzy structure following the sector nonlinearity approach [14].

$$
\begin{aligned}
& \Sigma_{1}\left\{\begin{array}{l}
\dot{\mathbf{x}}=\sum_{i=1}^{8} \mu_{i}(\mathbf{x}, \mathbf{z}, \mathbf{u})\left(\overline{\mathbf{A}}_{i} \mathrm{Z}+\overline{\mathbf{B}}_{i} \omega+\overline{\mathbf{D}}_{i} \mathbf{z}\right) \\
\mathbf{y}_{\mathbf{x}}=\overline{\mathbf{C}} \mathbf{x}
\end{array}\right. \\
& \Sigma_{2}\left\{\begin{array}{l}
\dot{\mathbf{z}}=\sum_{i=1}^{8} \sigma_{i}(\mathbf{x}, \mathbf{z}, \mathbf{u})\left(\breve{\mathbf{A}}_{j} \mathbf{X}+\breve{\mathbf{B}}_{j} \omega+\breve{\mathbf{D}}_{j} \mathbf{x}\right) \\
\mathbf{y}_{\mathbf{z}}=\breve{\mathbf{C}} \mathbf{z}
\end{array}\right.
\end{aligned}
$$

where $\mu_{i}(\hat{\mathbf{x}}, \mathbf{x}), i=1, \ldots, 8$ (respectively $\sigma_{i}(\mathbf{x}, \mathbf{z}, \mathbf{u}), i=$ $1, \ldots, 8)$ are the weighing functions satisfying the convex sum property stated in (17) with 8 being the number of submodels [15].

$$
\left\{\begin{array}{c}
0 \leqslant \mu_{i}(\mathbf{x}, \mathbf{z}, \mathbf{u}) \leqslant 1 \\
\sum_{i=1}^{8} \mu_{i}(\mathbf{x}, \mathbf{z}, \mathbf{u})=1
\end{array}\right.
$$

In our case each of the subsystems has 3 nonlinearities, which makes $2^{3}=8$ subsystem. The nonlinearities are defined for each interconnected model as:

$$
\begin{gathered}
\Sigma_{1}\left\{\begin{array}{l}
h_{1}=\frac{f_{v}}{f} \omega_{x}-\frac{f_{u}}{f} \omega_{y} \\
h_{2}=\chi v_{x}-\omega_{y} \\
h_{3}=f_{u} v_{z}
\end{array}\right. \\
\Sigma_{2}\left\{\begin{array}{l}
h_{1}=\frac{f_{v}}{f} \omega_{x}-\frac{f_{u}}{f} \omega_{y}+\chi v_{z} \\
h_{2}=-f v_{y} \\
h_{3}=\omega_{x}
\end{array}\right.
\end{gathered}
$$

After expressing the system in TS form, in the next section we address the interconnected observer design.

\section{OBSERVER DESIGN}

In this section, we address the problem of designing nonlinear observers for Takagi-Sugeno (TS) interconnected models with unmeasurable premise variable. The following assumptions are considered to ensure the observer existence.

Assumption 1: For a frozen values of the measured and unmeasured variables, we consider that:

1) The state vector and the system inputs are bounded.

2) Each subsystem is observable or at least detectable.

The proposed nonlinear interconnected observer includes two subsystems and it is given by:

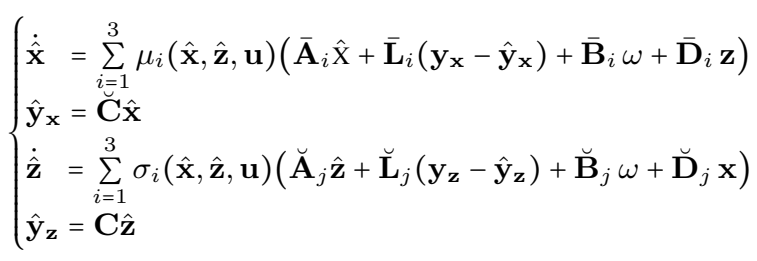

The estimated state for the first and the second sub-model are denoted respectively by $\hat{\mathbf{x}}$ and $\hat{\mathbf{z}}$.

Let us consider the state estimation errors: $\mathbf{e}_{\mathbf{x}}=\mathbf{x}-\hat{\mathbf{x}}$ and $\mathbf{e}_{\mathbf{z}}=\mathbf{z}-\hat{\mathbf{z}}$ The dynamics of the estimation errors are expressed by the following form:

$$
\left\{\begin{array}{l}
\dot{\mathbf{e}_{\mathbf{x}}}=\sum_{i=1}^{3} \mu_{i}(\hat{\mathbf{x}}, \hat{\mathbf{z}}, \mathbf{u})\left(\overline{\mathbf{\Phi}}_{i} \mathbf{e}_{\mathbf{x}}+\overline{\mathbf{D}}_{i} \mathbf{e}_{\mathbf{z}}+\Delta_{x}\right) \\
\dot{\mathbf{e}_{\mathbf{z}}}=\sum_{i=1}^{3} \sigma_{i}(\hat{\mathbf{x}}, \hat{\mathbf{z}}, \mathbf{u})\left(\breve{\boldsymbol{\Phi}}_{i} \mathbf{e}_{\mathbf{z}}+\breve{\mathbf{D}}_{j} \mathbf{e}_{\mathbf{x}}+\Delta_{z}\right)
\end{array}\right.
$$

where: $\overline{\mathbf{\Phi}}_{i}=\overline{\mathbf{A}}_{i}-\overline{\mathbf{L}}_{i} \overline{\mathbf{C}}, \breve{\mathbf{\Phi}}_{i}=\breve{\mathbf{A}}_{j}-\breve{\mathbf{L}}_{j} \breve{\mathbf{C}}, \Delta_{x}=\sum_{i=1}^{3}\left(\mu_{i}(\mathbf{x}, \mathbf{z}, \mathbf{u})-\right.$ $\left.\mu_{i}(\hat{\mathbf{x}}, \hat{\mathbf{z}}, \mathbf{u})\right) \overline{\mathbf{A}}_{i} \mathbf{x}$ and $\Delta_{z}=\sum_{i=1}^{3}\left(\mu_{i}(\mathbf{x}, \mathbf{z}, \mathbf{u})-\mu_{i}(\hat{\mathbf{x}}, \hat{\mathbf{z}}, \mathbf{u})\right) \breve{\mathbf{A}}_{i} \mathbf{z}$.

Taking into consideration the assumption 1 , since all the state vector and the inputs are bounded, we can say that $\Delta_{z}$ and $\Delta_{x}$ are also bounded and fulfill the following Lipschitz conditions:

$$
\begin{aligned}
& \Delta_{x}^{T} \Delta_{x}=\left\|\left(\Delta_{x}\right)\right\|^{2}<\alpha_{x}\|\hat{\mathrm{X}}-\mathrm{x}\|^{2}=\alpha_{x}\left\|\mathbf{e}_{\mathbf{x}}\right\|^{2} \\
& \Delta_{z}^{T} \Delta_{z}=\left\|\left(\Delta_{z}\right)\right\|^{2}<\alpha_{z}\left\|\hat{\mathbf{z}}-\mathbf{z}^{2}\right\|=\alpha_{z}\left\|\mathbf{e}_{\mathbf{z}}\right\|^{2}
\end{aligned}
$$

The Theorem stated below, establishes LMI constraints to guarantee the asymptotic stability of the estimation errors. 
Theorem 1:

The state estimation error converges asymptotically toward zero, if there exists positive definite symmetric matrices $\mathbf{P}, \mathbf{Q} \in \mathbb{R}^{2 \times 2}$, symmetric positive matrices $\Omega_{2}, \Omega_{1}, \mathbf{K}_{\mathbf{z}}, \mathbf{K}_{\mathbf{x}}, \mathbf{S}_{\mathbf{z}}, \mathbf{S}_{\mathbf{x}} \in \mathbb{R}^{2 \times 2}$ such that for a given scalars $\alpha_{1}, \alpha_{2}, \beta_{1}$ and $\beta_{2}$, the following LMIs constrains with pole clustering, defined in (25) and (24) are satisfied.

$$
\begin{gathered}
\left(\begin{array}{ccc}
-\Gamma_{i}+\mathbf{K}_{\mathbf{x}}+\Omega_{2} & \overline{\mathbf{D}}_{i}^{\top} \mathbf{P} & \mathbf{P} \\
\mathbf{P} \overline{\mathbf{D}}_{i} & -\Omega_{1} & 0 \\
\mathbf{P} & 0 & -\mathbf{S}_{\mathbf{x}}
\end{array}\right)<0 \\
\left(\begin{array}{cc}
\beta_{1} \mathbf{P} & \mathbf{P} \overline{\mathbf{A}}_{i}-\overline{\mathbf{W}}_{i} \overline{\mathbf{C}} \\
\overline{\mathbf{A}}_{i}^{\top} \mathbf{P}-\overline{\mathbf{C}} \top^{\top} \overline{\mathbf{W}} & \beta_{1} \mathbf{P}
\end{array}\right)>0
\end{gathered}
$$

$\overline{\mathbf{A}}_{i}^{\top} \mathbf{P}-\overline{\mathbf{C}}^{\top} \overline{\mathbf{W}}_{i}^{\top}+\mathbf{P} \overline{\mathbf{A}}_{i}-\overline{\mathbf{W}}_{i} \overline{\mathbf{C}}+2 \alpha_{1} \mathbf{P}<0 \quad i=1, \ldots, 8$

$$
\begin{gathered}
\left(\begin{array}{ccc}
-\breve{\Gamma}_{j}+\mathbf{K}_{\mathbf{z}}+\Omega_{1} & \breve{\mathbf{D}}_{j}^{\top} \mathbf{Q} & \mathbf{Q} \\
\mathbf{Q D}_{j} & -\Omega_{2} & 0 \\
\mathbf{Q} & 0 & -\mathbf{S}_{\mathbf{z}}
\end{array}\right)<0 \\
\left(\begin{array}{cc}
\beta_{1} \mathbf{Q} & \breve{\mathbf{A}}_{j}-\breve{\mathbf{W}}_{j} \breve{\mathbf{C}} \\
\breve{\mathbf{A}}_{j}^{\top} \mathbf{Q}-\breve{\mathbf{C}}^{\top} \breve{\mathbf{W}}_{j}^{\top} & \beta_{1} \mathbf{Q}
\end{array}\right)>0
\end{gathered}
$$

$\breve{\mathbf{A}}_{j}^{\top} \mathbf{Q}-\breve{\mathbf{C}}^{\top} \breve{\mathbf{W}}_{j}^{\top}+\mathbf{Q} \breve{\mathbf{A}}_{j}-\breve{\mathbf{W}}_{j} \breve{\mathbf{C}}+2 \alpha_{2} \mathbf{P}<0 \quad j=1, \ldots, 8$

where: $\bar{\Gamma}_{i}=\overline{\mathbf{A}}_{i}^{\top} \mathbf{P}-\overline{\mathbf{C}}^{\top} \overline{\mathbf{W}}_{i}^{\top}+\mathbf{P} \overline{\mathbf{A}}_{i}-\overline{\mathbf{W}}_{i} \overline{\mathbf{C}}$ and $\breve{\Gamma}_{j}=\breve{\mathbf{A}}_{j}^{\top} \mathbf{Q}-\breve{\mathbf{C}}^{\top} \breve{\mathbf{W}}_{j}^{\top}+\mathbf{Q} \breve{\mathbf{A}}_{j}-\breve{\mathbf{W}}_{j} \breve{\mathbf{C}}$

The resulting gains of the interconnected observer are given by: $\overline{\mathbf{L}}_{i}=\mathbf{P}^{-1} \overline{\mathbf{W}}_{i}, i=1, \ldots, 8$ and $\breve{\mathbf{L}}_{j}=\mathbf{Q}^{-1} \breve{\mathbf{W}}_{j}, j=1, \ldots, 8$

\section{Proof}

Considering the quadratic Lyapunov function given by:

$$
V=\mathbf{e}_{\mathbf{x}}^{\top} \mathbf{P} \mathbf{e}_{\mathbf{x}}+\mathbf{e}_{\mathbf{z}}^{\top} \mathbf{P} \mathbf{e}_{\mathbf{z}}
$$

where: $\mathbf{P}=\mathbf{P}^{T}>0$ and $\mathbf{Q}=\mathbf{Q}^{T}>0$

The time derivative of Lyapunov function yields:

$$
\begin{aligned}
\dot{V}(t)= & \dot{\mathbf{e}}_{\mathbf{x}}(t)^{\top} \mathbf{P} \mathbf{e}_{\mathbf{x}}(t)+\mathbf{e}_{\mathbf{x}}(t)^{\top} \mathbf{P} \dot{\mathbf{e}}_{x}(t)+ \\
& \dot{\mathbf{e}}_{\mathbf{z}}(t)^{\top} \mathbf{Q} \mathbf{e}_{\mathbf{z}}(t)+\mathbf{e}_{\mathbf{z}}(t)^{\top} \mathbf{Q} \dot{\mathbf{e}}_{\mathbf{z}}(t)
\end{aligned}
$$

Substituting the error dynamics equation (21), one obtains:

$$
\begin{aligned}
\dot{V}(t)= & \sum_{i=1}^{3} \mu_{i}(\hat{\mathbf{x}}, \hat{\mathbf{z}}, \mathbf{u})\left(\left(\overline{\mathbf{\Phi}}_{i} \mathbf{e}_{\mathbf{x}}+\overline{\mathbf{D}}_{i} \mathbf{e}_{\mathbf{z}}+\Delta_{x}\right)^{\top} \mathbf{P} \mathbf{e}_{\mathbf{x}}\right. \\
& \left.+\mathbf{e}_{\mathbf{x}}^{\top} \mathbf{P}\left(\overline{\mathbf{\Phi}}_{i} \mathbf{e}_{\mathbf{x}}+\overline{\mathbf{D}}_{i} \mathbf{e}_{\mathbf{z}}+\Delta_{x}\right)\right)+ \\
& \sum_{i=1}^{3} \sigma_{i}(\hat{\mathbf{x}}, \hat{\mathbf{z}}, \mathbf{u})\left(\left(\breve{\mathbf{\Phi}}_{j} \mathbf{e}_{\mathbf{z}}+\breve{\mathbf{D}}_{j} \mathbf{e}_{\mathbf{x}}+\Delta_{z}\right)^{\top} \mathbf{Q} \mathbf{e}_{\mathbf{z}}+\right. \\
& \left.\mathbf{e}_{\mathbf{z}}^{\top} \mathbf{Q}\left(\breve{\mathbf{\Phi}} \mathbf{e}_{\mathbf{z}}+\breve{\mathbf{D}}_{j} \mathbf{e}_{\mathbf{x}}+\Delta_{z}\right)\right)
\end{aligned}
$$

with: $\overline{\mathbf{\Phi}}_{i}=\overline{\mathbf{A}}_{i}-\overline{\mathbf{L}}_{i} \overline{\mathbf{C}}$ and $\breve{\mathbf{\Phi}}_{j}=\breve{\mathbf{A}}_{j}-\breve{\mathbf{L}}_{j} \breve{\mathbf{C}}$

Hence, the equation (28) is equivalent to:

$$
\begin{aligned}
\dot{V}(t)= & \sum_{i=1}^{8} \mu_{i}(\hat{\mathbf{x}}, \hat{\mathbf{z}}, \mathbf{u})\left(\mathbf{e}_{\mathbf{x}}^{\top}\left(\overline{\mathbf{\Phi}}_{i} \mathbf{P}+\mathbf{P} \overline{\mathbf{\Phi}}_{i}\right) \mathbf{e}_{\mathbf{x}}+\right. \\
& \left.\mathbf{e}_{\mathbf{z}}^{\top} \overline{\mathbf{D}}_{i} \mathbf{P} \mathbf{e}_{\mathbf{x}}+\mathbf{e}_{\mathbf{x}}^{\top} \mathbf{P} \overline{\mathbf{D}}_{i} \mathbf{e}_{\mathbf{z}}\right)+ \\
& \sum_{i=1}^{8} \sigma_{i}(\hat{\mathbf{x}}, \hat{\mathbf{z}}, \mathbf{u})\left(\mathbf{e}_{\mathbf{z}}^{\top}\left(\breve{\boldsymbol{\Phi}}_{j} \mathbf{Q}+\mathbf{Q} \breve{\boldsymbol{\Phi}}_{j}\right) \mathbf{e}_{\mathbf{z}}+\right. \\
& \left.\mathbf{e}_{\mathbf{x}}^{\top} \breve{\mathbf{D}}_{j} \mathbf{Q} \mathbf{e}_{\mathbf{z}}+\mathbf{e}_{\mathbf{z}}^{\top} \mathbf{Q} \breve{\mathbf{D}}_{i} \mathbf{e}_{\mathbf{x}}\right)+\Delta_{x}^{\top} \mathbf{P} \mathbf{e}_{\mathbf{x}}+ \\
& \left.\mathbf{e}_{\mathbf{x}}^{\top} \mathbf{P} \Delta_{x}+\Delta_{z}^{\top} \mathbf{Q} \mathbf{e}_{\mathbf{z}}+\mathbf{e}_{\mathbf{z}}^{\top} \mathbf{Q} \Delta_{z}\right)
\end{aligned}
$$

Using Lemma 1 the resulting inequalities hold:

$$
\begin{gathered}
\Delta_{x}^{\top} \mathbf{P} \mathbf{e}_{\mathbf{x}}+\mathbf{e}_{\mathbf{x}}^{\top} \mathbf{P} \Delta_{x} \leqslant \mathbf{e}_{\mathbf{x}}^{\top} \mathbf{P} \mathbf{H}_{\mathbf{x}} \mathbf{P} \mathbf{e}_{\mathbf{x}}+\Delta_{x}^{\top} \mathbf{H}_{\mathbf{x}}{ }^{-1} \Delta_{x} \\
\Delta_{z}^{\top} \mathbf{Q} \mathbf{e}_{\mathbf{z}}+\mathbf{e}_{\mathbf{z}}^{\top} \mathbf{Q} \Delta_{z} \leqslant \mathbf{e}_{\mathbf{z}}^{\top} \mathbf{Q} \mathbf{H}_{\mathbf{z}} \mathbf{Q} \mathbf{e}_{\mathbf{z}}+\Delta_{z}^{\top} \mathbf{H}_{\mathbf{z}}{ }^{-1} \Delta_{z} \\
\mathbf{e}_{\mathbf{z}}^{\top} \overline{\mathbf{D}}_{i} \mathbf{P} \mathbf{e}_{\mathbf{x}}+\mathbf{e}_{\mathbf{x}}^{\top} \mathbf{P} \overline{\mathbf{D}}_{i} \mathbf{e}_{\mathbf{z}} \leqslant \mathbf{P} \overline{\mathbf{D}}_{i} \mathbf{G}_{\mathbf{1}} \overline{\mathbf{D}}_{i}^{\top} \mathbf{P}+\mathbf{e}_{\mathbf{z}}{ }^{\top} \mathbf{G}_{\mathbf{1}}{ }^{-1} \mathbf{e}_{\mathbf{z}} \\
\mathbf{e}_{\mathbf{x}}^{\top} \breve{\mathbf{D}}_{j} \mathbf{Q} \mathbf{e}_{\mathbf{z}}+\mathbf{e}_{\mathbf{z}}^{\top} \mathbf{Q} \breve{\mathbf{D}}_{i} \mathbf{e}_{\mathbf{x}} \leqslant \mathbf{Q} \breve{\mathbf{D}}_{\mathbf{i}} \mathbf{G}_{\mathbf{2}} \breve{\mathbf{D}}_{\mathbf{j}}^{\top} \mathbf{Q}+\mathbf{e}_{\mathbf{x}}{ }^{\top} \mathbf{G}_{\mathbf{2}}{ }^{-1} \mathbf{e}_{\mathbf{x}}
\end{gathered}
$$

It yields:

$$
\begin{aligned}
\dot{V}(t) \leq & \sum_{i=1}^{8} \mu_{i}(\hat{\mathbf{x}}, \hat{\mathbf{z}}, \mathbf{u})\left(\mathbf{e}_{\mathbf{x}}^{\top}\left(\overline{\boldsymbol{\Gamma}}_{i}+\mathbf{P} \overline{\mathbf{D}}_{i} \mathbf{G}_{\mathbf{1}} \overline{\mathbf{D}}_{i}^{\top} \mathbf{P}\right)\right) \mathbf{e}_{\mathbf{x}}+ \\
& \mathbf{e}_{\mathbf{z}}{ }^{\top} \mathbf{G}_{\mathbf{1}}{ }^{-1} \mathbf{e}_{\mathbf{z}}+\sum_{i=1}^{8} \sigma_{i}(\hat{\mathbf{x}}, \hat{\mathbf{z}}, \mathbf{u})\left(\mathbf { e } _ { \mathbf { z } } { } ^ { \top } \left(\breve{\boldsymbol{\Gamma}}_{\mathbf{i}}+\right.\right. \\
& \left.\left.\left.\mathbf{Q D}_{\mathbf{i}} \mathbf{G}_{\mathbf{2}} \breve{\mathbf{D}}_{\mathbf{j}}^{\top} \mathbf{Q}\right) \mathbf{e}_{\mathbf{z}}\right)\right)+\mathbf{e}_{\mathbf{x}}^{\top} \mathbf{G}_{\mathbf{2}}{ }^{-1} \mathbf{e}_{\mathbf{x}}+\mathbf{e}_{\mathbf{x}}{ }^{\top} \mathbf{P} \mathbf{H}_{\mathbf{x}} \mathbf{P} \mathbf{e}_{\mathbf{x}}+ \\
& \Delta_{x}^{\top} \mathbf{H}_{\mathbf{x}}{ }^{-1} \Delta_{z}+\mathbf{e}_{\mathbf{z}}{ }^{\top} \mathbf{Q} \mathbf{H}_{\mathbf{z}} \mathbf{Q} \mathbf{e}_{\mathbf{z}}+\Delta_{z}^{\top} \mathbf{H}_{\mathbf{z}}{ }^{-1} \Delta_{z}
\end{aligned}
$$

with: $\bar{\Gamma}_{i}=\overline{\boldsymbol{\Phi}}_{i}^{\top} \mathbf{P}+\mathbf{P} \overline{\boldsymbol{\Phi}}_{i}$ and $\breve{\Gamma}_{j}=\breve{\boldsymbol{\Phi}}_{j}^{\top} \mathbf{Q}+\mathbf{Q} \breve{\boldsymbol{\Phi}}_{j}$. Taking into account the Lipschitz condition represented in the equations (23) and (22), then, the following inequality is satisfied:

$$
\begin{aligned}
\dot{V}(t) \leq & \sum_{i=1}^{8} \mu_{i}(\hat{\mathbf{x}}, \hat{\mathbf{z}}, \mathbf{u})\left(\mathbf { e } _ { \mathbf { x } } ^ { \top } \left(\overline{\boldsymbol{\Gamma}}_{i}+\mathbf{P} \overline{\mathbf{D}}_{i} \mathbf{G}_{\mathbf{1}} \overline{\mathbf{D}}_{i}^{\top} \mathbf{P}+\right.\right. \\
& \left.\left.\alpha_{x} \mathbf{H}_{x}^{-1}+\mathbf{P} \mathbf{H}_{x} \mathbf{P}+\mathbf{G}_{\mathbf{2}}{ }^{-1}\right) \mathbf{e}_{\mathbf{x}}\right)+ \\
& \sum_{i=1}^{8} \sigma_{i}(\hat{\mathbf{x}}, \hat{\mathbf{z}}, \mathbf{u})\left(\mathbf { e } _ { \mathbf { z } } { } ^ { \top } \left(\breve{\boldsymbol{\Gamma}}_{\mathbf{i}}+\mathbf{Q} \breve{\mathbf{D}}_{\mathbf{i}} \mathbf{G}_{\mathbf{2}} \breve{\mathbf{D}}_{\mathbf{j}}^{\top} \mathbf{Q}+\right.\right. \\
& \left.\left.\alpha_{z} \mathbf{H}_{z}^{-1}+\mathbf{Q} \mathbf{H}_{z} \mathbf{Q}+\mathbf{G}_{\mathbf{1}}{ }^{-1}\right) \mathbf{e}_{\mathbf{z}}\right)
\end{aligned}
$$

The derivative of Lyapunov function is definite negative $(\dot{V} \leq 0)$ if the following equations fulfilled:

$$
\begin{aligned}
& \sum_{i=1}^{8} \mu_{i}(\hat{\mathbf{x}}, \hat{\mathbf{z}}, \mathbf{u})\left(\mathbf { e } _ { \mathbf { x } } { } ^ { \top } \left(\overline{\boldsymbol{\Gamma}}_{i}+\mathbf{P} \overline{\mathbf{D}}_{i} \mathbf{G}_{\mathbf{1}} \overline{\mathbf{D}}_{i}^{\top} \mathbf{P}+\alpha_{x} \mathbf{H}_{x}^{-1}\right.\right. \\
& \left.\left.+\mathbf{P} \mathbf{H}_{x} \mathbf{P}+\mathbf{G}_{\mathbf{2}}{ }^{-1}\right) \mathbf{e}_{\mathbf{x}}\right)+\sum_{i=1}^{8} \sigma_{i}(\hat{\mathbf{x}}, \hat{\mathbf{z}}, \mathbf{u})\left(\mathbf { e } _ { \mathbf { z } } { } ^ { \top } \left(\breve{\boldsymbol{\Gamma}}_{\mathbf{i}}+\right.\right. \\
& \left.\left.\mathbf{Q D}_{\mathbf{i}} \mathbf{G}_{\mathbf{2}} \breve{\mathbf{D}}_{\mathbf{j}}^{\top} \mathbf{Q}+\alpha_{z} \mathbf{H}_{z}^{-1}+\mathbf{Q} \mathbf{H}_{z} \mathbf{Q}+\mathbf{G}_{\mathbf{1}}{ }^{-1}\right) \mathbf{e}_{\mathbf{z}}\right) \leq 0
\end{aligned}
$$

Thus, the inequality (37) is readily obtained:

$$
\left\{\begin{array}{l}
\overline{\boldsymbol{\Gamma}}_{i}+\mathbf{P} \overline{\mathbf{D}}_{i} \mathbf{G}_{\mathbf{1}} \overline{\mathbf{D}}_{\mathbf{i}}^{\top} \mathbf{P}+\alpha_{x} \mathbf{H}_{x}^{-1}+\mathbf{P} \mathbf{H}_{x} \mathbf{P}+\mathbf{G}_{\mathbf{2}}{ }^{-1} \leq 0 \\
\breve{\boldsymbol{\Gamma}}_{\mathbf{i}}+\breve{Q D}_{\mathbf{i}} \mathbf{G}_{\mathbf{2}} \breve{\mathbf{D}}_{\mathbf{j}}^{\top} \mathbf{Q}+\alpha_{z} \mathbf{H}_{z}^{-1}+\mathbf{Q} \mathbf{H}_{z} \mathbf{Q}+\mathbf{G}_{\mathbf{1}}{ }^{-1} \leq 0
\end{array}\right.
$$

In order to achieve solvable LMIs, the variables must appear linearly in the constraints derived. As a consequence, we adopt the following change of variable: $\Omega_{1}=\mathbf{G}_{\mathbf{1}}{ }^{-1}, \Omega_{2}=$ $\mathbf{G}_{\mathbf{2}}^{-1}, \quad \mathbf{K}_{\mathbf{z}}=\alpha_{z} \mathbf{H}_{z}^{-1}, \quad \mathbf{K}_{\mathbf{x}}=\alpha_{x} \mathbf{H}_{x}^{-1}, \quad \mathbf{S}_{\mathbf{z}}=\mathbf{H}_{z}^{-1}$ and $\mathbf{S}_{\mathbf{x}}=$ $\mathbf{H}_{x}^{-1}$

Then, using Schur complement lemma 2, the inequalities 37 can be expressed as:

$$
\begin{gathered}
\left(\begin{array}{ccc}
-\bar{\Gamma}_{i}+\mathbf{K}_{\mathbf{x}}+\Omega_{2} & \overline{\mathbf{D}}_{i}^{\top} \mathbf{P} & \mathbf{P} \\
\mathbf{P} \overline{\mathbf{D}}_{i} & -\Omega_{1} & 0 \\
\mathbf{P} & 0 & -\mathbf{S}_{\mathbf{x}}
\end{array}\right)<0 \\
\left(\begin{array}{ccc}
-\breve{\Gamma}_{j}+\mathbf{K}_{\mathbf{z}}+\Omega_{1} & \breve{\mathbf{D}}_{j}^{\top} \mathbf{Q} & \mathbf{Q} \\
\mathbf{Q D}_{j} & -\Omega_{2} & 0 \\
\mathbf{Q} & 0 & -\mathbf{S}_{\mathbf{z}}
\end{array}\right)<0
\end{gathered}
$$

To enhance the observer performance, and accelerate the rate convergence, the gain should be adjusted using pole clustering technique. The allocated region for pole placement 
is defined by the combination of a disk of radius $\beta$ centered at $(0,0)$ and the half plane delimited by the value $\alpha$ i.e:

$$
S(\alpha, \beta)=\{z \in \mathbb{C}, \mathfrak{R}(z)<-\alpha,|z|<\beta\}
$$

The pole clustering, considering two subsystems is governed by the following LMI formalism.

$$
\begin{aligned}
& \left(\begin{array}{cc}
\beta_{1} \mathbf{P} & \mathbf{P} \overline{\mathbf{A}}_{i}-\overline{\mathbf{W}}_{i} \overline{\mathbf{C}} \\
\overline{\mathbf{A}}_{i}^{\top} \mathbf{P}-\overline{\mathbf{C}}^{\top} \overline{\mathbf{W}} & \beta_{1} \mathbf{P}
\end{array}\right)>0 \\
& \overline{\mathbf{A}}_{i}^{\top} \mathbf{P}-\overline{\mathbf{C}}^{\top} \overline{\mathbf{W}}_{i}^{\top}+\mathbf{P} \overline{\mathbf{A}}_{i}-\overline{\mathbf{W}}_{i} \overline{\mathbf{C}}+2 \alpha_{1} \mathbf{P}<0 \quad i=1, \ldots, 3 \\
& \left(\begin{array}{cc}
\beta_{1} \mathbf{Q} & \mathbf{Q A}_{j}-\breve{\mathbf{W}}_{j} \breve{\mathbf{C}} \\
\breve{\mathbf{A}}_{j}^{\top} \mathbf{Q}-\breve{\mathbf{C}}^{\top} \breve{\mathbf{W}}_{j}^{\top} & \beta_{1} \mathbf{Q}
\end{array}\right)>0
\end{aligned}
$$$$
\breve{\mathbf{A}}_{j}^{\top} \mathbf{Q}-\breve{\mathbf{C}}^{\top} \breve{\mathbf{W}}_{j}^{\top}+\mathbf{Q} \breve{\mathbf{A}}_{j}-\breve{\mathbf{W}}_{j} \breve{\mathbf{C}}+2 \alpha_{2} \mathbf{P}<0 \quad j=1, \ldots, 3
$$

with $\alpha_{1}$ and $\beta_{1}$ are the region parameters of the first subsystem and $\beta_{2}$ and $\alpha_{2}$ are for the second subsystem.

Combining the LMIs constraints in 38, 39 and 40, we obtain the LMIs stated in the theorem 1 and this ends the proof.

\section{SIMULATION RESULTS}

Simulations are conducted in this section to test the performance of the observer taking into consideration two cases: a constant and a varying camera focal length. An image of an AprilTag, taken from MathWorks website, is warped using homography to create a sequence of images for both cases in order to evaluate the stability and precision of the observer. The homography is calculated and applied to every point in the image to generate different scenes, at a rate of $100 \mathrm{fps}$, comprising the AprilTag marker. Each dataset simulating a camera with a resolution of $2016 \times 1512$ pixels.

a) Case 1: considering a constant focal length: For the first simulation, we consider the constant focal length $f=1000$. The detected feature point is selected to be the point of the edge of one of the tag markers in the image as shown in Fig. ?? with the blue dot. The camera calibration matrix is set to:

$$
\mathbf{K}=\left(\begin{array}{ccc}
1000 & 0 & 1008 \\
0 & 1000 & 756 \\
0 & 0 & 1
\end{array}\right)
$$

The camera linear and angular velocities are given by:

$$
\begin{gathered}
v_{x}=0, v_{y}=-0.0005 t, v_{z}=-0.05 e^{-0.02 t}, \\
\omega_{x}=0.02, \omega_{y}=-0.07 \sin \left(\frac{\pi}{10} t\right), \omega_{z}=0.01
\end{gathered}
$$

The initial conditions of the interconnected observer are: $\hat{\mathbf{x}}=(253,200)^{\top}$ and $\hat{\mathbf{z}}=(10,2)^{\top}$.

Fig 2 reports the evolution of the real and estimated depth information during the simulation. And Fig. 3 depicts the behavior of the real and estimated focal length. The figures shows a fast convergence rate (around 3 seconds) of the estimated states to the real ones. And that is basically due to adjusting the interconnected observer gains using pole clustering (as represented in equations 39 and 40).

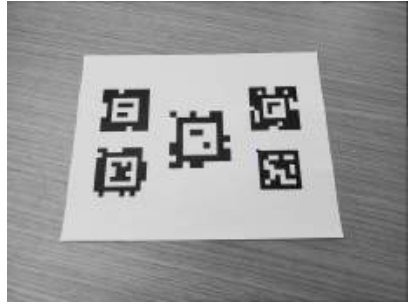

(a)

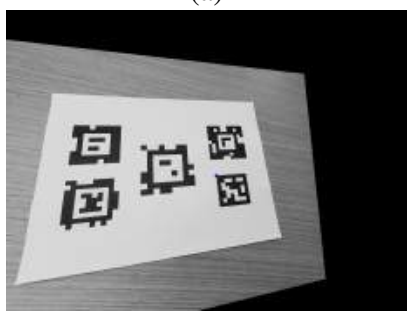

(c)

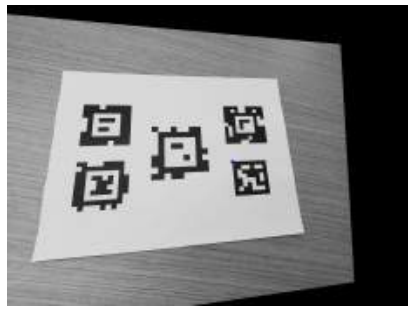

(b)

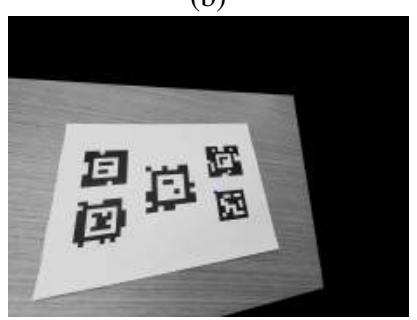

(d)
Fig. 1: (a) is the initial image, (b) image at $t=1 \mathrm{~s}$, (c) image at $t=3 \mathrm{~s}$ and (d) image at $t=6 \mathrm{~s}$ for the first case.

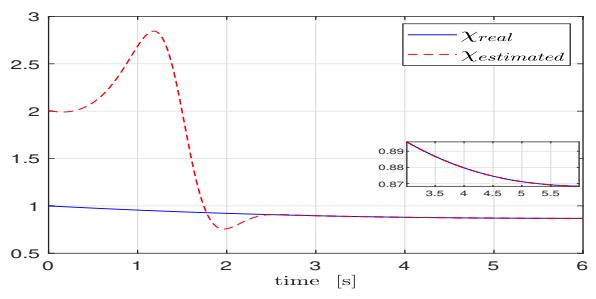

Fig. 2: Real (solid bleu line) and estimated (dashed red line) depth information $(\chi)$ for the first generated set of images

b) Case 2: considering a varying focal length: In order to show the observer performance, we analyse the case of a varying focal length The camera calibration matrix at $t=0$ is selected as:

$$
\mathbf{K}=\left(\begin{array}{ccc}
800 & 0 & 1008 \\
0 & 800 & 756 \\
0 & 0 & 1
\end{array}\right)
$$

The generated linear and angular velocities of the camera are given by:

$$
\begin{aligned}
& v_{x}=-0.02 e^{-0.02 t}, \quad v_{y}=-0.05 \sin \left(\frac{\pi}{5} t\right)-0.0005 t, \\
& v_{z}=-0.05-\cos \left(\frac{\pi}{5} t\right) \quad \omega_{x}=0.02 \cos \left(\frac{\pi}{4} t\right), \\
& \omega_{y}=0.06 \cos \left(\frac{\pi}{5} t\right), \quad \omega_{z}=0.01 e^{-0.05 t}
\end{aligned}
$$

The observer initial conditions are set to: $\hat{\mathbf{x}}=(-188,200)^{\top}$ and $\hat{\mathbf{z}}=(-200,2)^{\top}$.

Fig. 1 and Fig. 4 show the synthetic images of the first and second sequence at different times during the simulation where the tracked point is represented by the blue dot. In Fig. 6 the solid blue line shows the changes the real focal length across the views and it can be seen that the observer is still accurate even in case of varying focal length. The observer shows robust performance in estimating the actual values of the depth information $\chi$ and the camera focal length $f$ as it can be shown in Fig. 5 and Fig. 6. It's worth noting 


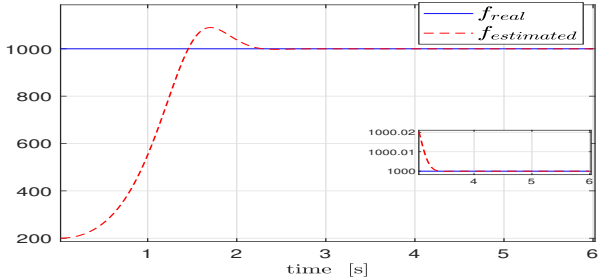

Fig. 3: Real (solid bleu line) and estimated (dashed red line) focal length $(f)$ for the first generated set of images

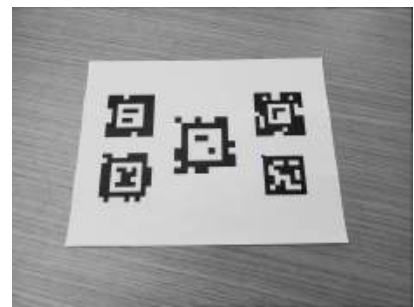

(a)

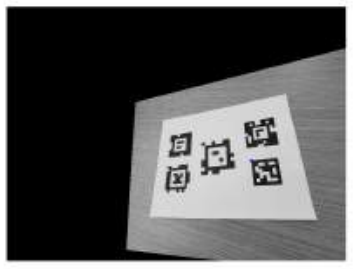

(c)

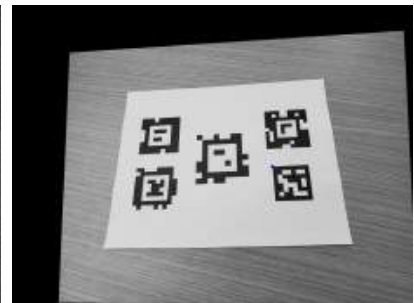

(b)

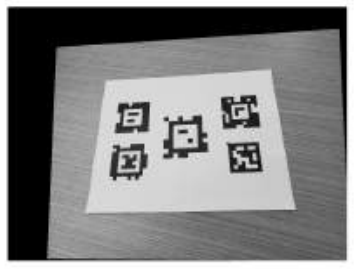

(d)
Fig. 4: (a) is the initial image, (b) image at $t=2 s$, (c) image at $t=5 \mathrm{~s}$ and (d) image at $t=10 \mathrm{~s}$ for the second case.

that the behaviour of the depth information $\chi$ in the case of varying focal length seems to be a little inconsistent when the focal length changes abruptly and this is directly related to the dynamics coupling between the two sub-models (the estimated state in one subsystem is the input for the other subsystem).

\section{CONCLUSIONS}

We have demonstrated, in this paper that the proposed interconnected observer produce very accurate results for the 3D structure estimation in case of partially calibrated camera. An interconnected observer design for nonlinear systems is investigated for qLPV systems with unmeasured premise variables, to estimate the 3D depth information and the camera focal length. In order to reduce the conservativeness of the LMIs, the considered system is obtained using a dynamic extension of a vision camera system and splited into two submodels. Necessary and sufficient conditions are provided to ensure the existence of the observer. The convergence of the estimation error is investigated based on Lyapunov theory. The observer gains are obtained by solving an LMI feasibility problem. Finally, simulations are carried out using synthetic data to validate the proposed observer. The presented approach gives an interesting solution for the $3 \mathrm{D}$ reconstruction using a partially calibrated camera.

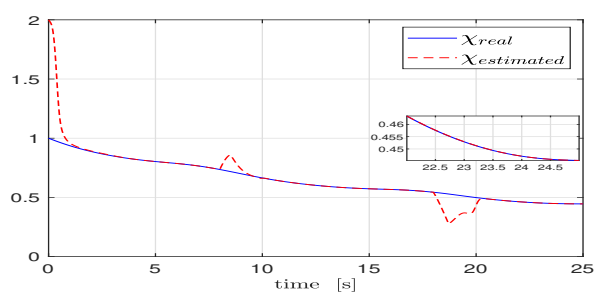

Fig. 5: Real (solid bleu line) and estimated (dashed red line)depth information $(\chi)$ for the second generated set of images

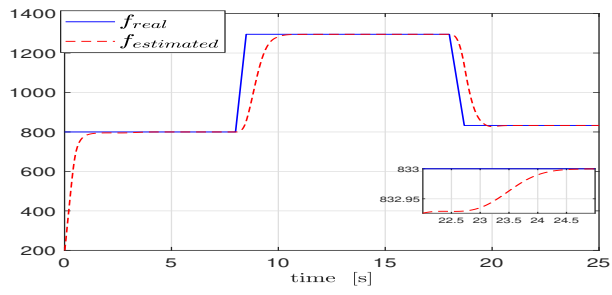

Fig. 6: Real (solid bleu line) and estimated (dashed red line) focal length $(f)$ for the second generated set of images

\section{REFERENCES}

[1] Kano, H., Ghosh, B. K., \& Kanai, H. (2001). Single camera based motion and shape estimation using extended Kalman filtering. Mathematical and Computer Modelling, 34(5-6), 511-525.

[2] Paramanand, C., \& Rajagopalan, A. N. (2011). Depth from motion and optical blur with an unscented Kalman filter. IEEE Transactions on Image Processing, 21(5), 2798-2811.

[3] Spica, R., \& Giordano, P. R. (2013, December). A framework for active estimation: Application to structure from motion. In 52nd IEEE conference on decision and control (pp. 7647-7653). IEEE.

[4] Mateus, A., Tahri, O., \& Miraldo, P. (2018, October). Active structurefrom-motion for $3 \mathrm{~d}$ straight lines. In 2018 IEEE/RSJ International Conference on Intelligent Robots and Systems (IROS) (pp. 58195825). IEEE.

[5] Spica, R., Giordano, P. R., \& Chaumette, F. (2014). Active structure from motion: Application to point, sphere, and cylinder. IEEE Transactions on Robotics, 30(6), 1499-1513.

[6] Spica, R., Giordano, P. R., \& Chaumette, F. (2015, May). Plane estimation by active vision from point features and image moments. In 2015 IEEE International Conference on Robotics and Automation (ICRA) (pp. 6003-6010). IEEE.

[7] Abraham, S., \& Förstner, W. (1998). Calibration errors in structure from motion. In Mustererkennung 1998 (pp. 117-124). Springer, Berlin, Heidelberg.

[8] Benyoucef, R., Nehaoua, L., Hadj-Abdelkader, H., \& Arioui, H. (2019, December). Depth Estimation for a Point Feature: Structure from motion \& Stability Analysis. In 2019 IEEE 58th Conference on Decision and Control (CDC) (pp. 3991-3996). IEEE.

[9] Grossmann, E., \& Santos-Victor, J. (2000). Uncertainty analysis of 3D reconstruction from uncalibrated views. Image and Vision Computing, 18(9), 685-696.

[10] Probst, T., Paudel, D. P., Chhatkuli, A., \& Van Gool, L. (2018). Incremental non-rigid structure-from-motion with unknown focal length. In Proceedings of the European Conference on Computer Vision (ECCV) (pp. 756-771).

[11] Bujnak, M., Kukelova, Z., \& Pajdla, T. (2009, September). 3d reconstruction from image collections with a single known focal length. In 2009 IEEE 12th International Conference on Computer Vision (pp. 1803-1810). IEEE.

[12] Chiuso, A., Favaro, P., Jin, H., \& Soatto, S. (2002). Structure from motion causally integrated over time. IEEE transactions on pattern analysis and machine intelligence, 24(4), 523-535. 
[13] Azarbayejani, A., \& Pentland, A. P. (1995). Recursive estimation of motion, structure, and focal length. IEEE Transactions on Pattern Analysis and Machine Intelligence, 17(6), 562-575.

[14] Ohtake, H., Tanaka, K., \& Wang, H. O. (2003). Fuzzy modeling via sector nonlinearity concept. Integrated Computer-Aided Engineering, 10(4), 333-341.

[15] Rotondo, D., Puig, V., Nejjari, F., \& Witczak, M. (2015). Automated generation and comparison of Takagi-Sugeno and polytopic quasiLPV models. Fuzzy Sets and Systems, 277, 44-64. 